\title{
Invalidity of the Ehrenfest theorem in the computation of high-order-harmonic generation within the strong-field approximation
}

\author{
C. Granados ${ }^{1}$ and L. Plaja ${ }^{1,2}$ \\ ${ }^{1}$ Departamento de Física Aplicada, Universidad de Salamanca, E-37008 Salamanca, Spain \\ ${ }^{2}$ Grupo de Investigación en Óptica Extrema, Universidad de Salamanca, E-37008 Salamanca, Spain
}

(Received 26 March 2012; published 2 May 2012)

\begin{abstract}
It is known that the strong-field approximations commonly used in models for computing high-order-harmonic generation invalidate the Ehrenfest theorem. Therefore, the time derivative of the atomic dipole or of the dipole velocity does not correspond to the dipole acceleration. We study the consequences of this invalidation for the quantitative evaluation of high-order-harmonic spectra in hydrogen at different wavelengths and intensities. As a main result, we propose a form for the time derivative of the kinetic momentum that allows a quantitatively accurate computation of the acceleration spectra using the dipole-velocity matrix elements.
\end{abstract}

DOI: 10.1103/PhysRevA.85.053403

PACS number(s): $32.80 . \mathrm{Wr}, 42.65 . \mathrm{Ky}$

\section{INTRODUCTION}

Intense-laser-matter interaction provides a privileged playground to test our understanding of quantum dynamics beyond the perturbative regime. The continuous developments in Chirped pulse amplification (CPA) amplification during recent decades have led to a mature technology available worldwide, permitting experiments with intense lasers in small laboratories and with moderate investment. Therefore, this field provides a scenario in which theory and experiments can be contrasted routinely. From the theoretical viewpoint, intense-laser-matter interaction is an attractive field as the form of the interaction Hamiltonian is simple, as a result of the dipole approximation. The simplicity of the interaction permits a focus on the problem of solving the nonperturbative dynamics, the derivation of models, and the gaining of intuition into the fundamental processes involved.

There are two main strategies for solving the dynamics of electrons, initially bounded in an atom or molecule and interacting with an intense laser field: the exact numerical solution of the time-dependent Schrödinger equation (TDSE) and the use of approximated models. In many cases, as when studying harmonic generation, the fundamental processes involve a single active electron. In this situation, although the exact integration of the TDSE is feasible in medium-sized computers, models play a fundamental role in speeding up calculations and providing the physical background of the processes involved. Among them, some of the most successful approaches are based on the Keldysh-Faisal-Reiss theory [1,3], which in this regime is equivalent to the strong-field approximation (SFA). Some refinements for high-order-harmonic generation (HHG) include also ionization rates computed with other approaches, such as, for instance, Perelomov, Popov and Terentiev theory (PPT theory) [4]. In this theory [5,6], the atomic electron is first tunnel-ionized from the atom, dwells in the continuum acquiring kinetic energy from the electromagnetic field, and finally releases it in the form of high-energy radiation upon rescattering with the parent ion. SFA models are known to provide reasonable predictions of the details of high-order-harmonic generation [7] and therefore they are extensively used with a combination of propagation codes to describe the radiation response of macroscopic targets, in order to reproduce typical experimental situations.

The coherent emission of the quantum atomic dipole can be computed according to the classical Larmor formula [8]

$$
P(t)=\frac{2}{3} \frac{q^{2}}{c^{3}}|\langle\hat{\mathbf{a}}(t)\rangle|^{2},
$$

where $q$ is the electron charge, $c$ is the velocity of light, and $\langle\hat{\mathbf{a}}(t)\rangle$ is the mean value of the acceleration. Since, assuming the dipole approximation $\hat{\mathbf{a}}(t)=(q / m)\left[-\nabla \hat{V}_{c}+\mathbf{E}(t)\right]$ [9], this quantity requires a precise knowledge of the electron's binding potential $V_{c}$. Therefore, it can be evaluated exactly only for the case of hydrogen. For many-electron atoms, and especially for molecules, the approximated forms for the gradient of the ionic potential are expressions from which it is not always possible to compute the matrix elements analytically. In these cases, it is common to resort to the Ehrenfest theorem and to compute the dipole acceleration as the first derivative of the dipole's velocity or the second derivative of the dipole moment, i.e., using the equivalence of

$$
\begin{gathered}
\mathbf{a}^{\nabla V_{c}}=-\frac{q}{m}\left\langle\nabla \hat{V}_{c}\right\rangle+\frac{q}{m} \mathbf{E}(t), \\
\mathbf{a}^{d v}=\frac{q}{m} \frac{\partial}{\partial t}\langle\hat{\mathbf{p}}\rangle, \\
\mathbf{a}^{d d r}=q \frac{\partial^{2}}{\partial t^{2}}\langle\hat{\mathbf{r}}\rangle,
\end{gathered}
$$

where $\mathbf{E}(t)$ is the driving field, $\hat{\mathbf{p}}$ is the kinetic momentum and $\hat{\mathbf{r}}$ the charge's coordinate. However, we shall demonstrate that this equivalence does not hold in the frame of the SFA, as the validity of the Ehrenfest theorem is affected by this approximation.

\section{GENERAL EXPRESSION FOR THE MEAN VALUE OF THE DIPOLE OPERATORS IN THE STRONG-FIELD APPROXIMATION}

The evaluation of the mean values of the different dipole operators (acceleration, velocity, or dipole moment) in the SFA follows the same route; therefore we will address it for a general odd-parity operator $\hat{O}$, representing any of the particular choices in Eqs. (2)-(4). 
Assuming a single active electron, we shall take as a starting point the integral solution of the Schrödinger equation [10]

$$
|\psi(t)\rangle=\left|\psi_{A}(t)\right\rangle+\left|\psi_{I}(t)\right\rangle,
$$

with

$$
\begin{gathered}
\left|\psi_{A}(t)\right\rangle=i G_{A}^{+}\left(t, t_{0}\right)\left|\phi_{0}\right\rangle, \\
\left|\psi_{I}(t)\right\rangle=\frac{1}{\hbar} \int_{t_{0}}^{t} d t_{1}^{\prime} G^{+}\left(t, t_{1}\right) V_{I}\left(t_{1}\right)\left|\psi_{A}\left(t_{1}\right)\right\rangle,
\end{gathered}
$$

associated with the splitting of the Hamiltonian into two parts $H(t)=H_{A}+V_{I}(t), H_{A}$ being the field-free atomic Hamiltonian, and $V_{I}(t)$ describing the interaction with an electromagnetic wave. Here $G^{+}\left(t, t_{1}\right)$ and $G_{A}^{+}\left(t, t_{1}\right)$ correspond to the propagators for the Coulomb-free and field-free cases, respectively. The ket $\left|\phi_{0}\right\rangle$ represents the initial state, which is assumed to be a bound eigenstate of $H_{A}$. Therefore, $\left|\psi_{A}(t)\right\rangle$ corresponds to the field-free evolution of the system and $\left|\psi_{I}(t)\right\rangle$ describes the perturbation by the driving field.

According to (5), the mean value of $\hat{O}$ can be written as

$$
\begin{aligned}
\langle\hat{O}\rangle= & \langle\psi(t)|\hat{O}| \psi(t)\rangle=\left[\left\langle\psi_{A}(t)|\hat{O}| \psi_{I}(t)\right\rangle+\text { c.c. }\right] \\
& +\left\langle\psi_{I}(t)|\hat{O}| \psi_{I}(t)\right\rangle,
\end{aligned}
$$

where the additional term $\left\langle\psi_{A}(t)|\hat{O}| \psi_{A}(t)\right\rangle$ is zero due to symmetry considerations. The SFA approximates (7) as

$$
\left|\psi_{I}(t)\right\rangle^{\mathrm{SFA}}=\frac{1}{\hbar} \int_{t_{0}}^{t} d t_{1}^{\prime} G_{F}^{+}\left(t, t_{1}\right) V_{I}\left(t_{1}\right)\left|\psi_{A}\left(t_{1}\right)\right\rangle,
$$

where $G_{F}$ is the propagator associated with the free-electron Hamiltonian $\hat{H}_{F}(t)=\hat{\mathbf{P}}^{2} / 2 m+V_{I}(t), \hat{\mathbf{P}}$ being the canonical momentum. Within this approximation, the perturbed wave function $\left|\psi_{I}(t)\right\rangle^{\text {SFA }}$ corresponds to a free electron moving under the sole influence of the electromagnetic field (i.e., neglecting the interaction with the residual ion). Accordingly, for the evaluation of the high-order-harmonic spectra, the term $\left\langle\psi_{I}(t)|\hat{O}| \psi_{I}(t)\right\rangle$ in Eq. (8) is commonly neglected as it describes the contribution of the free-free couplings to the mean dipole acceleration [11].

Combining Eqs. (6), (7) with (8), we obtain the SFA form of Eq. (8) to be used to compute high-order-harmonic generation:

$$
\langle\hat{O}\rangle^{\mathrm{SFA}}=\frac{1}{\hbar} \int_{t_{0}}^{t} d t_{1}^{\prime}\left\langle\psi_{A}(t)\left|\hat{O} G_{F}^{+}\left(t, t_{1}\right) V_{I}\left(t_{1}\right)\right| \psi_{A}\left(t_{1}\right)\right\rangle+\text { c.c. }
$$

\section{COMPARISON OF HARMONIC SPECTRA}

It is well known that the SFA breaks gauge invariance $[12,13]$. Therefore, to demonstrate the violation of the Ehrenfest theorem it is necessary to test it at least in the two most frequent gauges: length and velocity. Note that, for the reasons given below, $\mathbf{a}^{d v}$ is the common choice in the velocity gauge, while $\mathbf{a}^{d d r}$ is most used in the length gauge. Therefore, we shall compare $\mathbf{a}^{\nabla V_{c}}$ with $\mathbf{a}^{d v}$ or with $\mathbf{a}^{d d r}$ depending on the working gauge.

\section{A. Velocity gauge}

In the velocity gauge, the canonical momentum is a constant of motion for the free electron; therefore its eigenfunctions form a time-independent basis $\{|\mathbf{K}\rangle\}$, i.e.,

$$
\begin{gathered}
\hat{\mathbf{P}}|\mathbf{K}\rangle=\hbar \mathbf{K}|\mathbf{K}\rangle, \\
\hat{H}_{F}(t)|\mathbf{K}\rangle=\epsilon(\mathbf{K}, t)|\mathbf{K}\rangle,
\end{gathered}
$$

with $\epsilon(\mathbf{K}, t)=\left(\hbar^{2} / 2 m\right) K^{2}+V_{I}(\mathbf{K}, t)$ and $V_{I}(\mathbf{K}, t)=-(q \hbar / m c)$ $\mathbf{A}(t) \cdot \mathbf{K}+\left(q^{2} / 2 m c^{2}\right) A^{2}(t)$. Using this eigenbasis, and inserting the completeness relation $\int d \mathbf{K}|\mathbf{K}\rangle\langle\mathbf{K}|=\mathbb{1}$ in Eq. (10), we reach

$$
\begin{aligned}
\langle\hat{O}\rangle_{\text {vel }}^{\mathrm{SFA}}= & \frac{-i}{\hbar} \int d \mathbf{K} \int_{t_{0}}^{t} d t_{1}\left\langle\phi_{0}|\hat{O}| \mathbf{K}\right\rangle e^{-(i / \hbar) \int_{t_{1}}^{t}\left[\epsilon(\mathbf{K}, \tau)-\epsilon_{0}\right] d \tau} \\
& \times V_{I}(\mathbf{K}, t) \phi_{0}(\mathbf{K})+\text { c.c. }
\end{aligned}
$$

where $\phi_{0}(\mathbf{K})=\left\langle\mathbf{K} \mid \phi_{0}\right\rangle, \epsilon_{0}$ is the ground-state energy, and we have used

$$
\begin{gathered}
\left|\psi_{A}(t)\right\rangle=e^{\left.-(i / \hbar) \epsilon_{0}\left(t-t_{0}\right)\right]}\left|\phi_{0}\right\rangle, \\
i G_{F}^{+}\left(t, t_{1}\right)|\mathbf{K}\rangle=e^{-(i / \hbar) \int_{t_{1}}^{t} \epsilon(\mathbf{K}, \tau) d \tau}|\mathbf{K}\rangle .
\end{gathered}
$$

The SFA form of Eq. (2) can be written substituting $\hat{O}$ by $\nabla \hat{V}_{c}$ in Eq. (13), giving

$$
\begin{aligned}
\mathbf{a}_{\mathrm{vel}}^{\nabla V_{c}}(t)= & -\frac{i}{\hbar} \int d \mathbf{K} \int_{t_{0}}^{t} d t_{1} \mathbf{W}_{c}^{*}(\mathbf{K}) e^{-(i / \hbar) \int_{t_{1}}^{t}\left[\epsilon(\mathbf{K}, \tau)-\epsilon_{0}\right] d \tau} \\
& \times V_{I}\left(\mathbf{K}, t_{1}\right) \phi_{0}(\mathbf{K})+\text { c.c. },
\end{aligned}
$$

with $\mathbf{W}_{c}(\mathbf{K})=-(q / m)\left\langle\mathbf{K}\left|\nabla \hat{V}_{c}\right| \phi_{0}\right\rangle$ the matrix element of the Coulomb acceleration (we have dropped the term proportional to the fundamental field as it does not affect the high-order spectrum). On the other hand, the SFA form of Eq. (3) can be rewritten as

$$
\mathbf{a}_{\mathrm{vel}}^{d v}=\frac{q}{m} \frac{\partial}{\partial t}\langle\hat{\mathbf{P}}\rangle_{\mathrm{vel}}^{\mathrm{SFA}}+\frac{q}{m} \mathbf{E}(t),
$$

where we have expressed the kinetic momentum in terms of the canonical, $\hat{\mathbf{p}}=\hat{\mathbf{P}}-(q / m) \mathbf{A}(t)$.

The convenience of using $\mathbf{a}^{d v}$ in the velocity gauge is clear since $\hat{O}=\hat{P}$, and the matrix element $\left\langle\phi_{0}|\hat{O}| \mathbf{K}\right\rangle$ in Eq. (13) has the simple form $\mathbf{K} \phi_{0}(\mathbf{K})^{*}$; thus

$$
\begin{aligned}
\langle\hat{\mathbf{P}}\rangle_{\text {vel }}^{\text {SFA }}= & -\frac{i}{\hbar} \int d \mathbf{K} \int_{t_{0}}^{t} d t_{1} \hat{\mathbf{K}} e^{-(i / \hbar) \int_{t_{1}}^{t}\left[\epsilon(\mathbf{K}, \tau)-\epsilon_{0}\right] d \tau} \\
& \times V_{I}\left(\mathbf{K}, t_{1}\right)\left|\phi_{0}(\mathbf{K})\right|^{2}+\text { c.c. }
\end{aligned}
$$

Equations (16) and (18) can be integrated exactly using a Runge-Kutta algorithm [14].

Figure 1 plots the resulting spectra for the hydrogen atom computed in the velocity gauge, for a $\sin ^{2}$ envelope field of four cycles ( $3.75 \mathrm{fs}$ full width at half maximum) of a linearly polarized field of $1.57 \times 10^{14} \mathrm{~W} / \mathrm{cm}^{2}$ and wavelength 800 $\mathrm{nm}$. Although the compared spectra can be considered to have a remarkable qualitative agreement, there is a significant quantitative disagreement of more than one order of magnitude in the harmonic yields (a factor of 37 at the cutoff frequency). Note that we show results for an ultrashort pulse to have smooth spectra that will ease the comparison. Results for longer pulses follow a very similar behavior. 


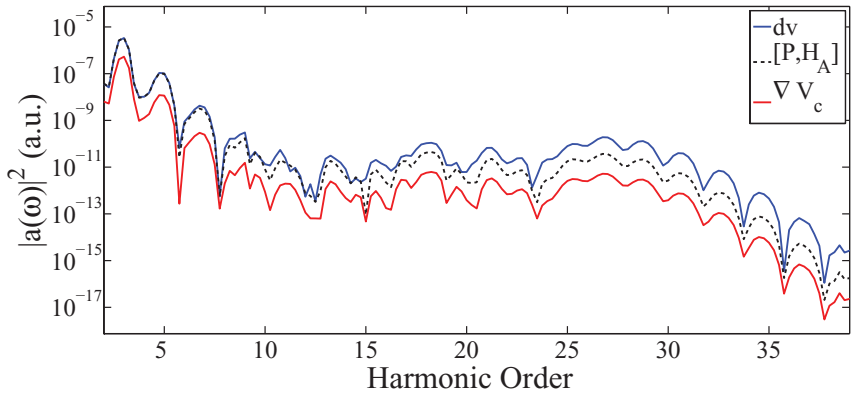

FIG. 1. (Color online) Comparison of SFA harmonic spectra computed in the velocity gauge for the hydrogen atom using Eq. (17), labeled as $\nabla V_{c}$ (red, lower line), and Eq. (16), labeled as $d v$ (blue, upper line). The fundamental field is a $\sin ^{2} 800 \mathrm{~nm}$ pulse of four cycles (total width) and intensity $1.57 \times 10^{14} \mathrm{~W} / \mathrm{cm}^{2}$. The black dashed line corresponds to the partially corrected spectrum of $\mathbf{a}_{\mathrm{vel}}^{\left[\hat{\mathbf{P}}, H_{A}\right]}$ derived in Sec. IV.

In order to show that the quantitative disagreement is not a mere constant factor, we have performed the same computations but for different wavelength and intensity. Figure 2 shows the comparisons of $\mathbf{a}_{\mathrm{vel}}^{d v}$ and $\mathbf{a}_{\mathrm{vel}}^{\nabla V_{c}}$ for a wavelength of $1600 \mathrm{~nm}$ and for two different intensities. The scale factors at the cutoff are 50 and 128, respectively. We find the dependence on the intensity of the mismatch introduced in using $\mathbf{a}^{d v}$ particularly relevant, as in a computation of harmonic generation from a macroscopic target, the driving field has a spatial profile with different intensities at the different target points.
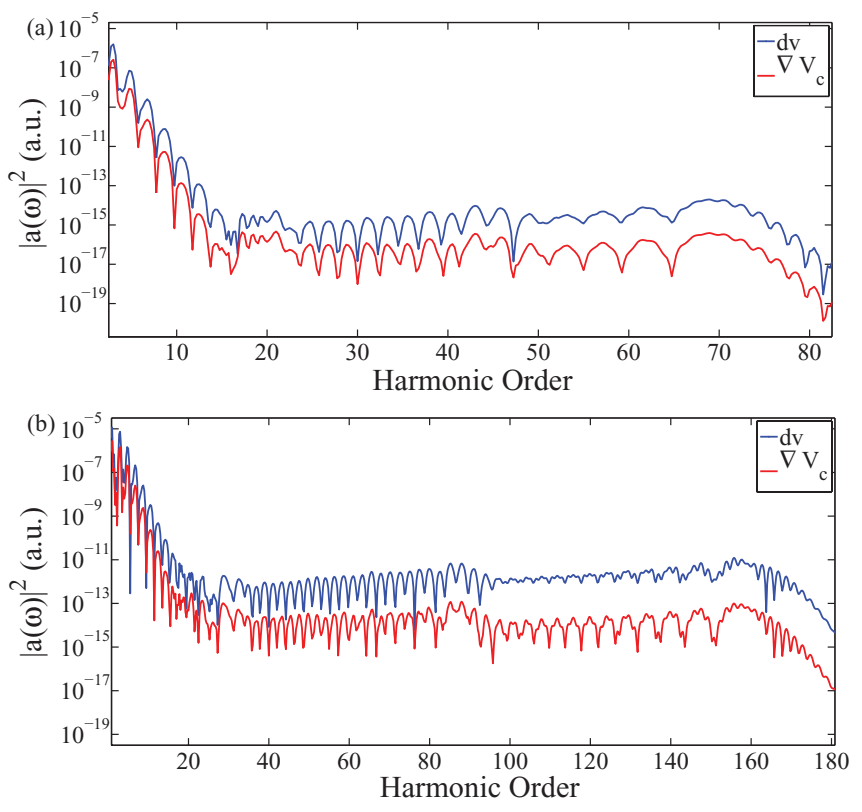

FIG. 2. (Color online) Comparison of SFA harmonic spectra computed in the velocity gauge for the hydrogen atom interacting with a four-cycle pulse (total width) $1600 \mathrm{~nm}$ laser field, for intensities (a) $5.6 \times 10^{13} \mathrm{~W} / \mathrm{cm}^{2}$ and (b) $1.57 \times 10^{14} \mathrm{~W} / \mathrm{cm}^{2}$. Labels refer to Eq. (17), labeled as $\nabla V_{c}$ (red, lower line) and Eq. (16), labeled as $d v$ (blue, upper line).

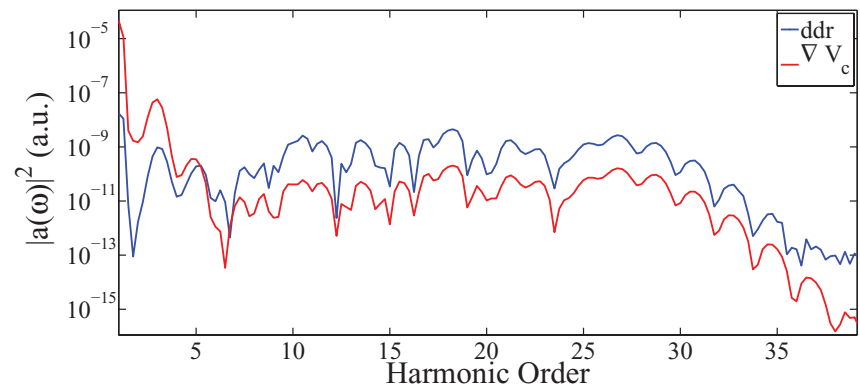

FIG. 3. (Color online) Harmonic spectra computed using the length gauge: result of Eqs. (4) and (20) is labeled as $d d r$ (blue, upper line), and that of Eq. (19) is labeled as $\nabla V_{c}$ (red, lower line). The fundamental field parameters are the same as in Fig. 1.

\section{B. Length gauge}

The same type of derivation as in the preceding section leads us, in the length gauge, to

$$
\begin{aligned}
\mathbf{a}_{\text {length }}^{\nabla V_{c}}(t)= & \frac{i}{\hbar} \int d \mathbf{K} \int_{t_{0}}^{t} d t_{1} \mathbf{W}_{c}^{*}[\mathbf{k}(t)] e^{-(i / \hbar) \int_{t_{1}}^{t}\left[\epsilon\left(\mathbf{K}, t_{1}\right)-\epsilon_{0}\right] d t_{1}} \\
& \times \mathbf{E}\left(t_{1}\right) \cdot \mathbf{d}\left[\mathbf{k}\left(t_{1}\right)\right]+\text { c.c. }
\end{aligned}
$$

with $\mathbf{k}(t)=\mathbf{K}-(q / \hbar m) \mathbf{A}(t)$ and $\mathbf{d}(\mathbf{k})=\left\langle\mathbf{k}|q \hat{\mathbf{r}}| \phi_{0}\right\rangle$ the matrix element of the dipole momentum. Also we have

$$
\begin{aligned}
\mathbf{r}_{\text {length }}(t)= & \frac{i}{q \hbar} \int d \mathbf{K} \int_{t_{0}}^{t} d t_{1} \mathbf{d}^{*}[\mathbf{k}(t)] e^{\left.-(i / \hbar) \int_{t_{1}}^{t} \in(\mathbf{K}, \tau)-\epsilon_{0}\right] d \tau} \\
& \times \mathbf{E}\left(t_{1}\right) \cdot \mathbf{d}\left[\mathbf{k}\left(t_{1}\right)\right]+\text { c.c. }
\end{aligned}
$$

to be used in Eq. (4) to evaluate $\mathbf{a}_{\text {length }}^{d d r}$.

The comparison between these two forms for the acceleration is found in Fig. 3, for the same parameters as in Fig. 1. We stress that the integration of Eqs. (19) and (20) is done exactly in this paper, as we have avoided the use of a saddlepoint method in order to perform our calculations as exactly as possible. We point out, anyway, that our checks of the saddle-point approximation using the present laser parameters have been positive. The conclusion for the length gauge is the same as previously: there is a considerable quantitative disagreement between the two forms of the acceleration.

\section{DISCUSSION}

The overriding of the Ehrenfest theorem when implementing the SFA has already been noticed by some authors. For instance, in Ref. [15] it is recognized that the time differentiation of the SFA wave function is not a proper procedure. Also, for the case of molecules, the structure itself of the harmonic spectrum is different if the acceleration is computed using (4), (3), or (2) [16-18]. On the other hand, it is also known that the HHG yields computed from the SFA have to be corrected to approach quantitatively the exact results of the Schrödinger equation. However, some recent SFA treatments [14] demonstrate that a simple extension to the standard SFA treatment leads to quantitatively accurate results for the harmonic yields computed using $\mathbf{a}_{\mathrm{vel}}^{\nabla V_{c}}$. Therefore it is clear that, if one wants to retain quantitative accuracy and has no exact information about the nature of the Coulomb potential, the question of whether the simpler forms (4) or (3) 
can be used within the SFA as a substitution for (2) constitutes a central issue.

The above results confirm the conclusions of the studies mentioned above, i.e., the inconsistency of the Ehrenfest theorem in the SFA. The main aim of this paper is, therefore, to propose a form for the time derivative of the kinetic momentum that should be of help in developing quantitative SFA HHG computations without a precise knowledge of the form of the Coulomb potential of the residual ion.

We shall now concentrate on the velocity gauge, and derive an alternative expression to $\mathbf{a}_{\mathrm{vel}}^{d v}$ that better approximates $\mathbf{a}_{\mathrm{vel}}^{\nabla V_{c}}$. The interest of this form is that it retains the same simplicity in implementation as $\mathbf{a}_{\mathrm{vel}}^{d v}$, as it depends only on the matrix elements of the momentum operator, while noticeably improving the agreement. To do this, we shall start from $\mathbf{a}_{\mathrm{vel}}^{\nabla V_{c}}$ in Eq. (2) and substitute $-\nabla \hat{V}_{c}$ by the equivalent conmutator $(1 / i \hbar)\left[\hat{\mathbf{P}}, \hat{H}_{A}\right]$. Assuming the SFA, we define the acceleration as

$$
\mathbf{a}_{\mathrm{vel}}^{\left[\hat{\mathbf{P}}, H_{A}\right]}(t)=\frac{q}{i \hbar m}\left\langle\left[\hat{\mathbf{P}}, \hat{H}_{A}\right]\right\rangle^{\mathrm{SFA}}+\frac{q}{m} \mathbf{E}(t) .
$$

Using (5) and (8) in the SFA, we have

$$
\begin{aligned}
\left\langle\left[\hat{\mathbf{P}}, \hat{H}_{A}\right]\right\rangle^{\mathrm{SFA}} & =\left\langle\psi_{A}(t)\left|\left[\hat{\mathbf{P}}, \hat{H}_{A}\right]\right| \psi_{I}(t)\right\rangle^{\mathrm{SFA}}+\text { c.c. } \\
& =\left\langle\psi_{A}(t)\left|\left(\hat{T}-\epsilon_{0}\right) \hat{\mathbf{P}}\right| \psi_{I}(t)\right\rangle^{\mathrm{SFA}}+\text { c.c. },
\end{aligned}
$$

where $\hat{T}=\hat{P}^{2} / 2 m$. Note that $\hat{H}_{A}\left|\psi_{I}(t)\right\rangle^{\mathrm{SFA}}=T\left|\psi_{I}(t)\right\rangle^{\mathrm{SFA}}$ as $\left|\psi_{I}(t)\right\rangle^{\mathrm{SFA}}$ corresponds to a free electron. Upon substitution in Eq. (13), the final form for $\mathbf{a}_{\mathrm{vel}}^{\left[\hat{\mathbf{P}}, H_{A}\right]}(t)$ is the same as $\mathbf{a}_{\mathrm{vel}}^{\nabla V_{c}}$ in Eq. (2), but with the corresponding matrix elements of $\nabla \hat{V}_{c}$ replaced by those of the conmutator (22); therefore

$$
\begin{aligned}
\mathbf{a}_{\mathrm{vel}}^{\left[\hat{\mathbf{P}}, H_{A}\right]}(t)= & \int d \mathbf{K} \int_{t_{0}}^{t} d t_{1} \mathbf{C}(\mathbf{K}) e^{-(i / \hbar) \int_{t_{1}}^{t}\left[\epsilon(\mathbf{K}, \tau)-\epsilon_{0}\right] d \tau} \\
& \times V_{I}\left(\mathbf{K}, t_{1}\right) \phi_{0}(\mathbf{K})+\text { c.c. },
\end{aligned}
$$

with $\mathbf{C}(\mathbf{K})=(1 / i \hbar)\left(K^{2} / 2 m-\epsilon_{0}\right) \mathbf{K}$.

The results for the spectrum of $\mathbf{a}_{\mathrm{vel}}^{\left[\hat{\mathbf{P}}, H_{A}\right]}$ compared to and $\mathbf{a}_{\mathrm{vel}}^{d v}$ and $\mathbf{a}_{\mathrm{vel}}^{\nabla V_{c}}$ have already been shown in Fig. 1. Note that, besides a slight improvement of the qualitative agreement, $\mathbf{a}_{\mathrm{vel}}^{\left[\hat{\mathbf{P}}, H_{A}\right]}$ is also quantitatively closer to $\mathbf{a}_{\mathrm{vel}}^{\nabla V_{c}}$ than is $\mathbf{a}_{\mathrm{vel}}^{d v}$. However, as the disagreement is still large, it is also apparent that the substitution of the time derivative leading to $\mathbf{a}_{\mathrm{vel}}^{\left[\hat{\mathbf{P}}, H_{A}\right]}$ solves the problem only partially. This is not entirely a surprise, since a term similar to $\mathbf{C}(\mathbf{K})$ has already been proposed in Ref. [15] as an alternative to the time derivative, although in the length gauge, giving a quantitative mismatch of the same order as here. In fact, we have found that the remaining inaccuracy of $\mathbf{a}_{\mathrm{vel}}^{\left[\hat{\mathbf{P}}, H_{A}\right]}$ (as shown in Fig. 1) can be traced back to the SFA, as it neglects the ionic potential and, therefore, the change in kinetic energy when the electron is near the ion, where the recombination occurs.

Let us develop a correction to this, based on a simplified picture. To do this, we shall consider two spatial regions (see Fig. 4): region I close to the ion and region II far from the ion. In region I we shall assume the Coulomb potential the relevant interaction, with the simplified form of a square potential well with depth $\left\langle V_{c}\right\rangle$. The potential-well states can

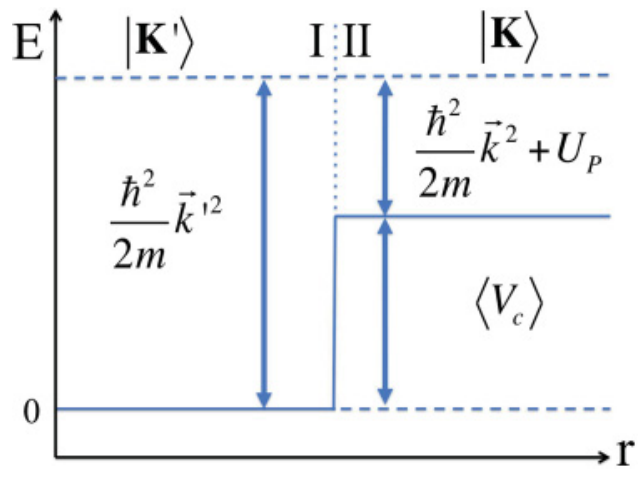

FIG. 4. (Color online) Scheme of the two spatial regions considered. Region I encloses electrons near the ion, where the Coulomb potential dominates, and region II electrons far from the ion, where the field force dominates.

be approximated by plane waves $\left|\mathbf{K}^{\prime}\right\rangle$ with energy

$$
E_{\mathrm{I}}=\frac{\hbar^{2} K^{\prime 2}}{2 m} \text {. }
$$

On the other hand, in region II, the field is the relevant interaction, and therefore we will assume free electrons $|\mathbf{K}\rangle$ with time-averaged energies

$$
E_{\mathrm{II}}=\frac{\hbar^{2} K^{2}}{2 m}+U_{p}-\left\langle V_{c}\right\rangle
$$

where $U_{p}$ is the ponderomotive energy acquired from the field and the term $-\left\langle V_{c}\right\rangle$ appears as we take the energy origin at the bottom of the potential well.

Setting (24) equal to (25), we have the following relation of the momentum of the plane waves of each region:

$$
\frac{\hbar^{2} K^{\prime 2}}{2 m}=\frac{\hbar^{2} K^{2}}{2 m}+U_{p}-\left\langle V_{c}\right\rangle .
$$

We now define a projection operator that describes the wave function in region II in the basis of region I,

$$
\hat{M} \simeq \int\left|\mathbf{K}^{\prime}\right\rangle\left\langle\mathbf{K}\left|d \mathbf{K}=\int\right| \mathbf{K}^{\prime}\right\rangle\langle\mathbf{K}| \eta(K) d \mathbf{K}^{\prime},
$$

where $\eta(K)=K / K^{\prime}$ appears because the volume element in the $|\mathbf{K}\rangle$ space, $K^{2} d K d \Omega$, does not coincide with that in $\left|\mathbf{K}^{\prime}\right\rangle$, $K^{\prime 2} d K^{\prime} d \Omega$, due to (26). Using (26) and taking $\left\langle V_{c}\right\rangle$ as the mean value of the Coulomb potential in the atomic ground state, $-2\left|\epsilon_{0}\right|$, we have

$$
\eta(K)=\left(1+\frac{U_{p}+2\left|\epsilon_{0}\right|}{\hbar^{2} K^{2} / 2 m}\right)^{-1 / 2} .
$$

We can, therefore, consider replacement of the momentum in Eq. (22) by the transformed form

$$
\hat{\mathbf{P}}^{\prime}=\hat{M}^{-1} \hat{\mathbf{P}} \hat{M}
$$

which leads to a modified rescattering coefficient that takes into account the Coulomb correction,

$$
\begin{aligned}
\mathbf{C}^{\prime}(\mathbf{K}) & =(1 / i \hbar)\left(K^{2} / 2 m-\epsilon_{0}\right) \mathbf{K}^{\prime} \eta^{2}(K) \\
& =(1 / i \hbar)\left(K^{2} / 2 m-\epsilon_{0}\right) \mathbf{K} \eta(K) .
\end{aligned}
$$



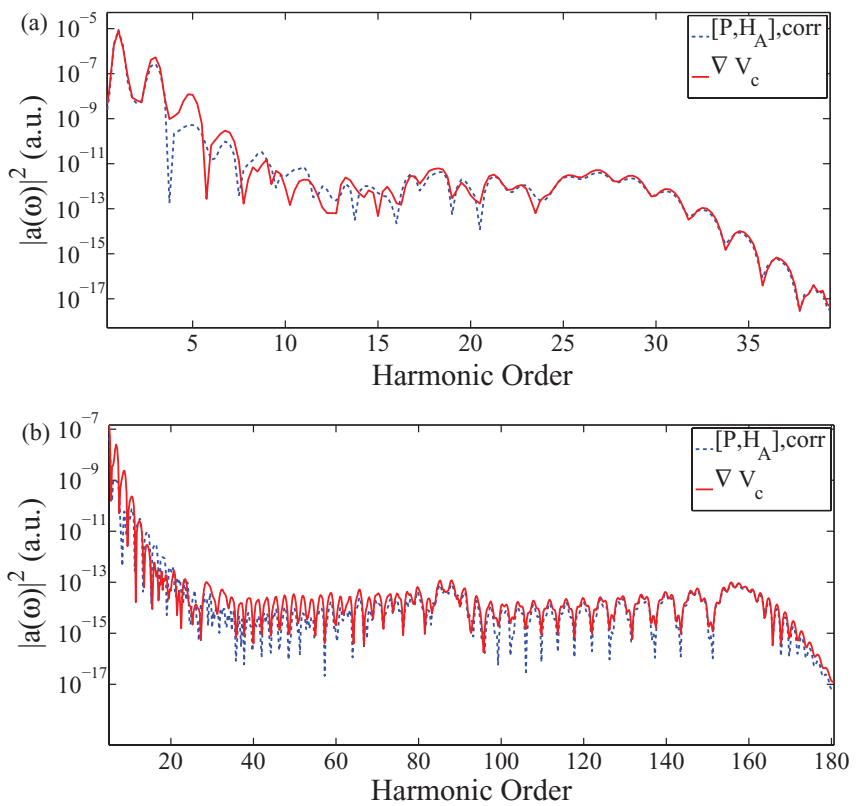

FIG. 5. (Color online) Comparison between the harmonic spectra using Eq. (31), labeled as $\left[P, H_{A}\right]$, corr (blue dashed line), and (16), labeled as $\nabla V_{c}$ (red line), for the same parameters used in (a) Fig. 1 and (b) Fig. 2(b).

Therefore, the corrected form of (23) reads

$$
\begin{aligned}
\mathbf{a}_{\mathrm{vel}}^{\left[\hat{\mathbf{P}}, H_{A}\right], \operatorname{corr}}(t)= & \int d \mathbf{K} \int_{t_{0}}^{t} d t_{1} \mathbf{C}^{\prime}(\mathbf{K}) e^{-(i / \hbar) \int_{t_{1}}^{t}\left[\epsilon(\mathbf{K}, \tau)-\epsilon_{0}\right] d \tau} \\
& \times V_{I}\left(\mathbf{K}, t_{1}\right)\left|\phi_{0}(\mathbf{K})\right|^{2}+\text { c.c. }
\end{aligned}
$$

To test our proposal (31) as an alternative to $\mathbf{a}_{\mathrm{vel}}^{\nabla V_{C}}$, we plot in Fig. 5(a) the high-order harmonic spectra for $\mathbf{a}_{\mathrm{vel}}^{\left[\hat{\mathbf{P}}, H_{A}\right], \text { corr }}$ and $\mathbf{a}_{\mathrm{vel}}^{\nabla V_{C}}$ for the same laser parameters as in Fig 1. In addition, Fig. 5(b) shows the same comparison but for a different wavelength, corresponding to the case plotted in Fig 2(b). The quantitative agreement is notable, which is certainly remarkable in view of the simplicity in the derivation of the model we have employed. We should note also that the above correction has been proven to work solely in the velocity gauge. The trivial extension to the length gauge, which would consist in the replacement of the canonical momentum by the time-dependent kinetic momentum, leads to dipole-derived harmonic spectra that differ considerably from those calculated from the acceleration, both quantitatively and qualitatively.

\section{CONCLUSION}

It is widely recognized that the strong-field approximation is an invaluable tool for developing simplified models for the intense-field-atom interaction. However, it is also known that the model description depends on the particular implementation of the SFA, for instance on the gauge employed. In accordance with previous work, in this paper we have demonstrated that the SFA also affects the validity of the Ehrenfest theorem and, therefore, that the description of high-order-harmonic generation is not equivalent if computed through the direct calculation of the mean acceleration or from the time derivative of the dipole velocity or of the dipole moment. However, the computation of these two latter quantities is often more accessible and, hence, they are widely used. In this paper we have identified the source of the problem as twofold: on one hand, the time-derivative operator in the SFA seems to be less accurate than its Ehrenfest equivalent, the commutator with the Hamiltonian; on the other hand, it is necessary to implement a Coulomb correction to the momentum operator, to compensate the information loss in approximating the continuum states as plane waves. Accordingly, we propose a corrected form for the SFA acceleration that uses the momentum matrix elements and that reproduces with quantitative accuracy the result obtained using the acceleration matrix elements.

\section{ACKNOWLEDGMENTS}

We thank Enrique Conejero for useful criticisms and discussions. We acknowledge support from the Spanish Ministerio de Economía y Competitividad through the Consolider program SAUUL (Grant No. CSD2007-0013) and Research Project No. FIS2009-09522 and also from the Centro de Láseres Pulsados (CLPU), Salamanca.
[1] L. V. Keldysh, Zh. Eksp. Teor. Fiz. 47, 1945 (1964) [Sov. Phys. JETP 20, 1307 (1965)].

[2] F. H. M. Faisal, J. Phys. B 6, L89 (1973).

[3] H. R. Reiss, Phys. Rev. A 22, 1786 (1980).

[4] A. M. Perelomov, V. S. Popov, and M. V. Terentiev, Sov. Phys. JETP 23, 924 (1966).

[5] P. B. Corkum, Phys. Rev. Lett. 71, 1994 (1993).

[6] K. J. Schafer, B. Yang, L. F. Di Mauro, and K. C. Kulander, Phys. Rev. Lett. 70, 1599 (1993).

[7] M. Lewenstein, Ph. Balcou, M. Yu. Ivanov, A. L'Huillier, and P. B. Corkum, Phys. Rev. A 49, 2117 (1994).

[8] J. D. Jackson, Classical Electrodynamics (Wiley, New York, 1998).

[9] K. Burnett, V. C. Reed, J. Cooper, and P. L. Knight, Phys. Rev. A 45, 3347 (1992).
[10] W. Greiner, Quantum Electrodynamics (Springer, Berlin, 2003).

[11] W. Becker, A. Lohr, M. Kleber, and M. Lewenstein, Phys. Rev. A 56, 645 (1997).

[12] J. H. Bauer, J. Phys. B 41, 185003 (2008).

[13] D. Bauer, D. B. Milosevic, and W. Becker, Phys. Rev. A 72, 023415 (2005).

[14] J. A. Pérez-Hernández, L. Roso, and L. Plaja, Opt. Express 17, 9891 (2009).

[15] Ariel Gordon and Franz X. Kärtner, Phys. Rev. Lett. 95, 223901 (2005).

[16] G. L. Kamta and A. D. Bandrauk, Phys. Rev. A 71, 053407 (2005).

[17] C. C. Chirilă and M. Lein, J. Mod. Opt. 54, 1039 (2007).

[18] B. B. Augstein and C. Figueira de Morisson Faria, J. Mod. Opt. 58, 1173 (2011). 